\title{
Tromboprofilaxis en otorrinolaringología
}

\section{Thrombosis prophylaxis in otorhinolaryngology}

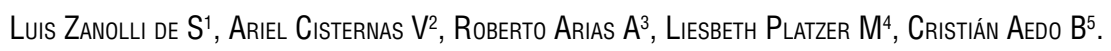

\begin{abstract}
RESUMEN
Introducción: La tromboprofilaxis es una indicación médica, muchas veces omitida, que tiene un muy alto nivel de evidencia que la avala. Sin embargo, no existen en el área de la otorrinolaringología ni en cirugía de cabeza y cuello, trabajos que objetiven su necesidad y su uso.

Objetivo: Evaluar la necesidad de tromboprofilaxis y la realidad de su uso en nuestra especialidad, comparando nuestros resultados con las recomendaciones internacionales.

Material y método: Se realizó una revisión retrospectiva de las fichas clínicas de los pacientes otorrinolaringológicos hospitalizados durante el mes de marzo de 2007 en los cinco centros formadores de posgrado de otorrinolaringología de Santiago de Chile. Se determinó si presentaban o no factores de riesgo para enfermedad tromboembólica (riesgo moderado, alto o muy alto), y si se usó o no la tromboprofilaxis en cada uno de los casos.

Resultados: Del universo de 545 pacientes 119 tenían factores de riesgo para enfermedad tromboembólica constituyendo el $21,8 \%$ de la muestra, $10,2 \%(n=56)$ pacientes presentaban riesgo moderado, 5,9\% $(n=32)$ alto y $5,7 \%(n=31)$ muy alto riesgo. Sólo en el 12,6\% de los pacientes con indicación, se utilizó tromboprofilaxis.

Discusión y conclusiones: Se evidenció que más del $20 \%$ de los pacientes tenían indicación de tromboprofilaxis, sin embargo, el uso de la profilaxis adecuada se realizó sólo en el 12,6\% de estos casos. La utilización de los protocolos de tromboprofilaxis constituyen una herramienta útil para mejorar los niveles de uso de esta medida mundialmente aceptada.

Palabras claves: Tromboprofilaxis, tromboembolismo, prevención en otorrinolaringología.
\end{abstract}

\section{ABSTRACT}

Introduction: Thrombosis prophylaxis is a medical indication, often times overlooked, backed by a considerable body of evidence. However, its need and use in the areas of otorhinolaryngology and head and neck surgery are still to be precised.

Aim: To assess the need for thrombosis prophylaxis and to document its use in ORL, comparing the results with the international recommendations for its use.

\footnotetext{
Médico Servicio de Otorrinolaringología, Hospital Barros Luco.

Médico Servicio de Otorrinolaringología, Hospital San Juan de Dios.

Médico Servicio de Otorrinolaringología, Hospital del Salvador.

Médico Servicio de Otorrinolaringología, Hospital Clínico de la Pontificia Universidad Católica de Chile.

Médico Servicio de Otorrinolaringología, Hospital Clínico de la Universidad de Chile.
} 
Material and Method: Retrospective review of medical records of ORL patients hospitalized during the month of March 2007 in the five centers that provide postgraduate ORL training in Santiago, Chile. The existence of thromboembolic disease risk factors (moderate, high or very high) and the use or not of thrombosis prophylaxis were documented.

Results: From a cohort of 545 patients, 119 presented risk factors for thrombosis (21.8\% of the sample); $10.2 \%(m=56)$ had moderate risk, $5.9 \% \quad(n=32)$ high risk and $5.7 \%(n=31)$ very high risk. Thrombosis prophylaxis was used in only $12.6 \%$ of the patients that had it indicated.

Discussion and Conclusion: This work demonstrate that more than $20 \%$ of the reviewed patients had an indication for thrombosis prophylaxis; however, only in $12.6 \%$ of cases adequate prophylaxis was used. Utilization of thrombosis prophylaxis protocols is a useful tool in order to improve the use of this widely accepted measure.

Key words: Thrombosis prophylaxis, thromboembolism, prevention in otorhinolaryngology.

\section{INTRODUCCIÓN}

La otorrinolaringología constituye una especialidad médico quirúrgica que involucra en su diario quehacer, la hospitalización de ambos tipos de pacientes, ya sea pacientes portadores de alguna condición médica que requiera hospitalización para su manejo, o bien, pacientes que requieran de una intervención quirúrgica como parte de su tratamiento. En ambos casos, debemos enfrentar a cabalidad cada una de las condiciones, patologías y/o riesgos asociados.

Muchas veces, y en forma errada, se atribuye la trombosis venosa profunda a especialidades médicas como la traumatología y la cirugía abdominal, sin considerar que los principales factores en el desarrollo de esta patología no son exclusivas de éstas. Es bien sabido, que la patogenia del tromboembolismo está fundamentalmente determinada por la estasia venosa y la hipercoagulabilidad, ambos factores frecuentemente concomitantes en los pacientes hospitalizados en los servicios clínicos de otorrinolaringología ${ }^{1}$ así como también en los sometidos a alguna cirugía de la esfera otorrinolaringológica.

También está ampliamente demostrado que el uso de tromboprofilaxis reduce la incidencia de trombosis venosa profunda y sus complicaciones, por lo que su indicación en casos de pacientes seleccionados se respalda con los más altos niveles de evidencia ${ }^{2-4}$. El 2001 en Estados Unidos la prevención del tromboembolismo fue considerada como el principal desafío en pacientes hospitali- zados $^{5}$. El Reino Unido en 2005 consideró al tromboembolismo como causante de 25.000 muertes prevenibles al año, siendo la mitad de ellas en pacientes hospitalizados ${ }^{6}$.

\section{OBJETIVO}

El objetivo primario de este trabajo es evaluar la necesidad de tromboprofilaxis en pacientes otorrinolaringológicos hospitalizados por causas médicas y/o quirúrgicas.

Como objetivos secundarios, se analizará la utilización de tromboprofilaxis en una muestra de los servicios de otorrinolaringología de Santiago de Chile. Al mismo tiempo, queremos difundir las pautas de uso de tromboprofilaxis.

\section{MATERIAL Y MÉTODO}

Se realizó un estudio multicéntrico, retrospectivo y descriptivo revisando el universo total de fichas

Tabla 1. Total pacientes, uso de profilaxis y $\%$ de uso

\begin{tabular}{|lrrr|}
\hline & Total & $\mathrm{n}$ & $\%$ \\
\hline Riesgo moderado & 56 & 5 & 8,9 \\
Riesgo alto & 32 & 0 & 0 \\
Riesgo muy alto & 31 & 10 & 32,2 \\
\hline Total & 119 & 15 & 12,6 \\
\hline
\end{tabular}


clínicas de los pacientes hospitalizados y/u operados durante el mes de marzo de 2007 en los servicios de otorrinolaringología de los 5 centros formadores de posgrado de otorrinolaringología de Santiago de Chile: Hospital Clínico de la Universidad de Chile, Hospi-

\section{Tabla 2. Factores de riesgo para pacientes} hospitalizados y quirúrgicos

\begin{tabular}{|ll}
\hline Factores de Riesgo para pacientes hospitalizados \\
\hline Condición & Infección aguda \\
& Falla cardiaca congestiva \\
& Infarto agudo miocárdico \\
& Enfermedad respiratoria aguda \\
& Accidente vascular encefálico \\
& Enfermedad reumatológica \\
& Enfermedad inflamatoria del intestino \\
Características & Tromboembolismo previo \\
& Ancianos (especialmente $>75$ años) \\
& Cirugía reciente o traumatismo \\
& Inmovilidad o paresias \\
& Obesidad (IMC mayor de 30) \\
& Catéter venoso central \\
& Estados trombofílicos \\
& Venas varicosas \\
& Terapia estrogénica
\end{tabular}

Factores de riesgo para pacientes quirúrgicos

- Cáncer como causa de cirugía

- Tiempo del procedimiento (mayor a $30 \mathrm{~min}$.)

- Trombosis venosa profunda previa

- Tabaquismo

- Edad mayor a 40 años

- Obesidad

\begin{tabular}{|ll|}
\hline Bajo riesgo & $\begin{array}{l}\text { Cirugía menor, en }<40 \text { años sin factores } \\
\text { de riesgo. }\end{array}$ \\
Moderado riesgo & Cirugía menor con un factor de riesgo 0, \\
Elto riesgo & Mayores de a 60 años sin factores de riesgo \\
Muy alto riesgo 0 & Cirugía en pacientes con múltiples facto- \\
& res de riesgo, ej. Mayores de 40 más \\
& cáncer más TVP previa. Cáncer como \\
& causa de cirugía, tiempo del procedimien- \\
& to (mayor a 30 min.), trombosis venosa \\
& profunda previa, tabaquismo, edad ma- \\
& yor a 40 años y obesidad.
\end{tabular}

tal Clínico de la Pontificia Universidad Católica, Hospital del Salvador, Hospital San Juan de Dios y Hospital Barros Luco Trudeau.

Se consignaron los antecedentes y las características clínicas de los pacientes, considerando los factores de riesgo que presentaban para desarrollar una enfermedad tromboembólica. Así, se categorizaron según su riesgo de presentarla en: riesgo moderado, riesgo alto y riesgo muy alto, en base a lo publicado por Geerts y cols. y Francis $^{1,7,8}$ (Tabla 2). Se determinó si se usó 0 no tromboprofilaxis y si ésta se ajustó a lo sugerido por las normas internacionales ${ }^{9}$ (Tabla 3 ).

\section{RESULTADOS}

En un universo de 545 pacientes hospitalizados por razones médico quirúrgicas en el mes de marzo 2007 en los 5 hospitales estudiados, un total de 119 pacientes presentaban factores de riesgo de enfermedad tromboembólica, constituyendo el $21,8 \%$ de la muestra. De ellos, 56 (10,2\%) pacientes presenta-

Tabla 3

\begin{tabular}{|l} 
Medicamentos y dosis para profilaxis de tromboembolismo \\
para pacientes médicos \\
Droga y Dosis \\
Heparina no fraccionada 5.000 U subcutánea c/8 horas. \\
Enoxaparina $40 \mathrm{mg}$ subcutánea una vez al día. \\
Dalteparina 5000 U subcutánea una vez al día. \\
Medicamentos y dosis para profilaxis de tromboembolismo \\
para pacientes quirúrgicos según riesgo \\
$\begin{array}{ll}\text { Bajo riesgo: } & \text { Movilización precoz y persistente. } \\
\text { Moderado riesgo: } & \text { Medias elásticas o compresión } \\
& \text { neumática intermitente. } \\
& \text { Heparina no fraccionada } 5000 \text { uni- } \\
& \text { dades subcutáneas } 2 \text { veces al día. } \\
\text { Alto riesgo y Muy Alto: } & \text { Heparina de bajo peso, Enoxaparina no fraccionada } 5000 \text { uni- } \\
& \text { dades subcutáneas } 3 \text { veces al día. } \\
& \text { Heparina de bajo peso, Enoxapa- } \\
& \text { rina } 40 \text { mg o Dalteparina } 5000 \mathrm{u} \\
& \text { día. } \\
& \text { Medias elásticadas y o compresión } \\
& \text { neumática intermitente. }\end{array}$
\end{tabular}


ban un riesgo moderado, $32(5,9 \%)$ tenían un riesgo alto y $31(5,7 \%)$ presentaban un riesgo muy alto.

El uso adecuado de tromboprofilaxis se realizó en 5 pacientes de los que tenían riesgo moderado, en el grupo de riesgo alto ningún paciente tuvo medida profiláctica y en el grupo de alto riesgo sólo se utilizaron medidas tromboprofilácticas en 10 pacientes.

El uso global de tromboprofilaxis fue en 15 pacientes de 119 constituyendo el 12,6\% (Tabla 1).

\section{DISCUSIÓN}

El uso de la tromboprofilaxis es una intervención que a pesar de su alto nivel de evidencia no se ha difundido en nuestro medio, existe una escasa conciencia de sus complicaciones y altos costos asociados. En nuestro estudio colaborativo queda en evidencia que existe un gran número de pacientes que requieren tromboprofilaxis, ya que presentan condiciones constitucionales, además de sus patologías asociadas, que determinan riesgos.

Creemos que el uso de pautas o protocolos de consenso en los servicios determinarán una mejor adherencia al uso de la tromboprofilaxis.

En la actualidad existe consenso que la tromboprofilaxis es una medida mundialmente aceptada y que el riesgo de sangrado agregado no contraindica su uso y que si por condiciones propias de la cirugía el sangrado es de alto riesgo se deben realizar las medidas mecánicas ${ }^{8}$. En el año 2005 el "Health Select Committee" del Reino Unido publicó que la tromboprofilaxis no había sido implementada en forma eficiente y que sólo el $20 \%$ de los pacientes elegibles para la medida la habían recibido adecuadamente ${ }^{6}$. En el caso de los pacientes de riesgo moderado existe evidencia que el uso de medias de compresión graduada disminuye los eventos de trombosis venosa profunda ${ }^{10}$ por lo que todos los protocolos los incluyen a partir de los pacientes de riesgo moderado en adelante.

\section{BIBLIOGRAFÍA}

1. Francis CW. Prophylaxis for thromboembolism in hospitalized medical patients. N Engl J Med 2007; 356 (14): 1438-44.

2. Collins R, Scrimgeor A, Yusuk $S$, Peto R. Reduction in fatal pulmonary embolism and venous thrombosis by perioperative administration of subcutaneous heparin. Overview of results of randomised trials in general, orthopaedic and urologic surgery. N Engl $J$ Med 1988; 318: 1162-73.

3. Mismetti P, Laporte S, Darmon Jy, Buchmuller A, Decousus $H$. Meta-analysis of low molecular weight heparin in the prevention of venous thromboembolism in general surgery. $\mathrm{Br} \mathrm{J}$ Surg 2001; 88: 913-30.

4. Cohen at, Davidson BL, Gallus as, Lassen MR, Prins MH, TomkowskI W; ARTEMIS Investigators. Efficacy and safety of fondaparinux for the prevention of venous thromboembolism in older acute medical patients: randomised placebo controlled trial. BMJ 2006; 332: 325-9.

5. Agency for Healthcare Research and Quality. Making health care safer. A critical analysis of patient safety practices: summary. Evidence Report/Technology Assessment Number 43. Rockville, MD: AHRQ, July.

6. Fitzmaurice DA, Murray E. Thomboprophylaxis for adults in hospital. BMJ 2007; 334: 1017-8.

7. Geerts WH, Heit JA, Clagett GP, et al. Chest 2001; 119 (suppl 1): 132-75.

8. Agnelu G. Prevention of venous thromboembolism in surgical patients. Circulation 2004; 110 (24 Suppl 1): 4-12.

9. William H Geerts, Graham F Pineo, John A Heit, et AL. Prevention of Venous Thromboembolism. The Seventh ACCP Conference on Antithrombotic and Thrombolytic Therapy. CHEST 2004; 126 : 338-400.

10. Agu 0, Hamilton G, Baker D. Graduated compression stockings in the prevention of the thromboembolism. BMJ 1999; 86: 9921004.

Dirección: Dr. Luis Zanolli de S.

Av. José Miguel Carrera 3204, San Miguel. Santiago, Chile.

E mail: luiszanolli@yahoo.com 